\title{
The impact of the MARINET initiative on the development of Marine Renewable Energy
}

\author{
Maisondieu Christophe ${ }^{1,{ }^{*}}$, Healy Mark ${ }^{2}$
}

${ }^{1}$ IFREMER, Z.I. Pointe du Diable, CS 10070, 29280 Plouzané, France

${ }^{2}$ Beaufort Research - HMRC, University College Cork, Pouladuff Road, Togher, Cork, Ireland

* Corresponding author: Christophe Maisondieu, Tel.: +332 98224733 ;

email address : christophe.maisondieu@ifremer.fr

\begin{abstract}
:
Marine Renewable Energy Conversion systems comprise wave energy and tidal stream converters as well as offshore-wind turbines for electrical generation. These technologies are currently at different stages of development but are mostly at the pre-commercial stage and require research to be undertaken at a series of scales along the path to commercialization. However each of these technologies also needs specific research infrastructures in order to conduct this research. The aim of the MARINET initiative is to coordinate research and development at all scales (small models through to prototype scales, from laboratories through to open sea tests) and to allow access for researchers and developers to infrastructures which are not available universally in Europe, including test facilities for components such as power take-off systems, grid integration, moorings and environmental monitoring so as to ensure a focusing of activities in this area. The initiative offers researchers and developers access to 45 research facilities as well as to the associated network of expertise at all scales in Offshore Marine Renewable Energy technology research and development.

The aim of this paper is to present this MARINET initiative that was started in 2011, bringing together a network of 29 partners spread across twelve countries. Details of the MARINET Transnational Access (TA) program are presented, for which over 260 applications were received throughout the 5 official calls for proposals. In particular, statistics on applications and completed projects are presented which provide an overview of the global development progress of the different offshore renewable energy conversion technologies at a European level. It also provides a good overview of the current research activity, as well as evidence of the requirement for specialised research facilities, in this burgeoning field.
\end{abstract}




\section{Highlights}

- The Transnational Access programme : a key element of the MARINET initiative 256 proposals providing a good overview of the stage of development of the Marine Renewable Energy industry in Europe

Keywords: Marine Renewable Energy, Research Infrastructures, Testing Facilities, Technology Readiness Level, Structured Development Plan

\section{Introduction}

Among the various Marine Renewable Energy sources generally considered for power extraction and electrical generation, waves, tidal streams and off-shore wind are the most widely and thoroughly investigated. Numerous conversion systems for these three sources of energy have been studied and designed over previous decades. These technologies are currently at different stages of development but are mostly at the pre-commercial stage and still require research to be undertaken at a series of scales along the path to commercialization. However each one also needs specific specialized research infrastructures. The Marine Renewables Infrastructure Network, MARINET, was formed to coordinate research and development at all scales (small models through to prototype scales, from laboratories 
through to open sea tests) based on the Technology Readiness Level (TRL) of the technology and to allow access for researchers and developers to infrastructures which are not available universally in Europe. The consortium brings together a large network of infrastructures such as wave tanks and tidal flumes, but also including test facilities for components such as power take-off systems, grid integration, moorings and environmental tests. The aim is to ensure a focusing of research activities and resources in these various areas, as well as coordinating the expertise of personnel who are specialists in this offshore marine renewable energy sector, at all scales of marine technology research and development.

A key element of the MARINET initiative, in addition to the programmes for improvement and standardisation of testing methods, is the Transnational Access (TA) programme. This offers free of charge public access to the research facilities in order to support the development of technologies or research concepts from laboratories through to open sea tests. This TA programme, decomposed in five calls for proposals organised over a period of approximately three years, enabled a large number of developers and research groups across Europe to conduct c. 165 testing programmes in dedicated facilities in order to assess and improve their research concept or the efficiency of their technologies, at various Technology Readiness Levels (TRL).

Cross-analysis of the statistics related to access to infrastructures during this Transnational Access programme provides a good overview of the stage of development of the Marine Renewable Energy industry in Europe. It also highlights some of the constraints encountered by developers proceeding through the multi-staged process of development of these technologies for wave, tidal and off-shore wind energy conversion.

Details of the MARINET Transnational Access programme initiative are presented in section two of this paper. Access statistics are analysed and discussed in section three, whilst benefits and limitations of the Transnational Access programme are discussed in the last section.

\section{The MARINET Initiative}

The MARINET initiative was started in March 2011. It brings together a network of 29 partners, spread across twelve countries to offer researchers and developers access to 45 research facilities as well as a network of expertise at all scales of Offshore Marine Renewable Energy (MRE) technology research and development. The aim is to streamline the testing path in order to accelerate the development and commercial deployment of marine renewable energy technologies [1].

The common theme straddling the two main MARINET elements of:

1) a joint sector approach to the improvement and development of infrastructure capabilities and services for the benefit of the industry, and

2) free-of-charge access to world-class infrastructures through the Transnational Access programme,

is the definition and adoption of common test and development standards in order to facilitate the streamlined and efficient progression of test devices from small-scale facilities through to larger-scale facilities and open-sea sites. This is achieved primarily by:

- adopting a standardized approach to testing and development across all infrastructures and scales, using the IEA-OES marine energy Structured Development Plan which is aligned to the well-known concept of Technology Readiness Levels and 
- facilitating networking and training for industry and research in the form of User Workshops and short-courses to provide opportunities for training, collaboration, joint ventures and familiarization with the Structured Development approach.

This paper focusses specifically on the $2^{\text {nd }}$ element of the MARINET initiative, the Transnational Access programme.

\subsection{The Transnational Access programme}

Among the 29 partners of the MARINET consortium, 24 institutions across ten countries offer developers access to 45 research facilities through the Transnational Access (TA) programme, whereby companies and research groups can benefit from periods of free-of-charge access to these test facilities to conduct technology testing and development. The Transnational Access (i.e. to facilities located outside the users' home country) can be availed of to test devices at any scale in areas such as wave energy, tidal energy, offshore-wind energy \& environmental data or to conduct sub-systems tests on cross-cutting areas such as power take-off systems, electrical grid integration, materials or moorings.

\subsubsection{Facilities classification}

Facilities involved in the Transnational Access programme are classified by MARINET according to:

a) the scale of testing they can accommodate, ranging from small scale laboratory through to large scale offshore testing sites and

b) the focus group area to which they are related, namely waves, tidal currents, off-shore wind and cross-cutting.

Specific infrastructures such as data bases are classified as large-scale sites. Table 1 hereafter provides an overview of this classification, outlining the number of facilities available in each scale and focus group. The number of infrastructures categorised in this table is larger than the 45 reference number as some infrastructures can be used for research in overalpping focus areas, such as wave tanks for instance which can be used for testing both wave converters and off-shore wind floats.

In this classification, laboratories are classified as facilities offering testing capacities in a dedicated controlled environment such as wave tanks, current flumes or wind tunnels, whilst testing sites are classified as monitored sites located in the natural environment in which the natural forcing, namely, wind, waves and current, cannot be controlled.

Designating these focus areas also allows User Groups (the visiting researchers accessing an infrastructure) the possibility of benefiting from the knowledge of experts in each field along the access path, from the selection process through to the completion of the trials programme. Projects are assessed by specialists in the particular field and the testing programme is conducted in an infrastructure managed by an infrastructure team having identified expertise in the specific field.

All kinds of technologies for wave energy conversion are considered in the wave research focus group, whether they are adapted to coastal areas or the open sea and including gravity based or floating structures. In a similar way, the tidal focus group is dedicated to technologies for tidal current energy extraction, not just limited to turbines but considering all tidal current energy technologies. The scope of the offshore-wind focus group covers the research for floating offshore wind production and also includes environmental databases (as they are mostly related to wind resource). Finally, the cross- 
cutting group covers the widest range of research activities including materials, moorings, power takeoff devices or grid integration.

\begin{tabular}{|c|c|c|c|c|}
\hline \multirow{2}{*}{$\begin{array}{l}\text { SCALE } \\
\downarrow\end{array}$} & \multicolumn{4}{|c|}{$\leftarrow$ FOCUS AREA $\rightarrow$} \\
\hline & $\begin{array}{l}\text { Wave } \\
\text { Energy }\end{array}$ & $\begin{array}{c}\text { Tidal } \\
\text { Energy }\end{array}$ & $\begin{array}{l}\text { Offshore-Wind Energy and } \\
\text { Environmental Data }\end{array}$ & $\begin{array}{c}\text { Cross-Cutting } \\
\text { Areas }\end{array}$ \\
\hline Small Scale Lab & 6 & 5 & 3 & 5 \\
\hline Large Scale Lab & 7 & 4 & 2 & 5 \\
\hline Medium Scale Site & 3 & 3 & 3 & 1 \\
\hline Large Scale Site & 2 & 1 & 8 & 3 \\
\hline
\end{tabular}

Table 1: Classification of Infrastructures available for Transnational Access

\subsubsection{Applications}

The objective of Transnational Access is to offer users free-of-charge access to the research and testing infrastructues of the MARINET network. As there are limitations in the availability of each infrastructure, as well as time limits imposed by the EU specifications for the programme, it was necessary to implement a procedure covering the whole duration of the project which allowed an optimized use of the access time. Therefore, five periods of access were organised over the duration of the project, each of them having a 6 to 12 months duration. A selection procedure was organised for each of these access periods, starting with a call for proposals scheduled at least 3 months ahead of the starting date of the access period. The selection procedure itself was divided into four steps. Eligibility of applications according to MARINET rules and conditions was first verified. The main criterion here being that applicants and their group must be based in a country other than the country where the legal entity operating the applied-for MARINET infrastructure is established. The basis of this EU requirement is to encourage transnational access, a key objective of the EU. This key requirement has an impact on the user's choice of infrastructure.

Subsequent stages of the selection process include an assessment of the technical feasibility of the proposal by the infrastructure manager of the applied-for facility, together with an evaluation by external experts providing judgment on the scientific content and relevance of the outcome of the proposal. Final allocations of infrastructure time are then made by the MARINET User Selection Board.

An important element taken into account in the judgment of the quality of the proposals is the suitability of the requested facility to the particular Stage of development of the project, according to the IEA-OES Structured Development Plan.

\subsubsection{Structured Development Plan linked to TRLs}

The Structured Development Plan sets out the development Stages required for a marine renewable energy concept to achieve commercial reality.

Over the years, devices have been developed in an ad-hoc fashion and as a result, many failures have occurred in the misguided rush to get full-scale devices into the sea. This has resulted in significant investment losses, a slow rate of technology development and credibility issues for the sector. In order to attempt a standardised approach to marine energy technology development, an international Structured Development Plan has been produced. This concept, aligned to the proven steps of Technology Readiness Levels originally established by NASA and widely used by many engineering 
research establishments, has gained acceptance worldwide through the International Energy Agency's intergovernmental collaboration 'Ocean Energy Systems Implementing Agreement' (OES) [2], [3] and through the European FP7 project EQUIMAR [4]. It is now used as the main marine energy development and assessment method in the US and Europe.

The protocols for wave, tidal and offshore-wind energy differ slightly, but the basic concept of following a Structured Development Plan is the same. An example of the wave energy Structured Development Plan is given below. In all of the Stages, there is a requirement for testing of physical models. The MARINET facilities follow and offer support at all stages of the Plan, and users are required to show that they are following this Plan.

\begin{tabular}{|c|c|c|c|}
\hline $\begin{array}{c}\text { Structured } \\
\text { Development Plan } \\
\text { Stage }\end{array}$ & TRL & Objectives & Scale Guide \\
\hline 1 & $1-3$ & Design validation/Device optimization & $1: 100-1: 25$ \\
\hline 2 & 4 & Performance verification/Components testing & $1: 25-1: 10$ \\
\hline 3 & $5-6$ & $\begin{array}{c}\text { Operational converter sea trials } \\
\text { Energy production assessment }\end{array}$ & $1: 5-1: 2$ \\
\hline 4 & $7-8$ & $\begin{array}{c}\text { Full size production Technical deployment } \\
\text { Pre-commercial unit }\end{array}$ & $1: 2-1: 1$ \\
\hline 5 & 9 & $\begin{array}{c}\text { Final commercial unit - Economic deployment } \\
\text { Small array trials - Grid issues }\end{array}$ & $1: 1$ \\
\hline
\end{tabular}

Table 2: Structured Development Plan aligned to TRLs

The number of facilities in each scale-class, together with the total number of eligible proposals received in each scale-class, is given in table 3 hereafter. The last column of the table indicates the ratio of proposals received in each scale-class as a percentage of the total number (256) of eligible projects.

This table clearly shows that with about $82 \%$ of the total number of proposals, facilities classified as small and large labs were in the highest demand.

\begin{tabular}{|l|c|c|c|}
\hline Scale class & Number of facilities & Number of proposals & $\begin{array}{l}\text { Ratio of proposals } \\
(\boldsymbol{\%})\end{array}$ \\
\hline Small Lab & 14 & 89 & 34.8 \\
\hline Large Lab & 13 & 121 & 47.3 \\
\hline Medium-scale site & 5 & 22 & 8.6 \\
\hline Large-scale site & 13 & 24 & 9.4 \\
\hline
\end{tabular}

Table 3: Number of proposals per facility class

Over the two and a half years of effective operation of the Transnational Access programme with the five calls for proposals that were organised, a total of 256 eligible proposals were received. From these, 159 projects have been successful at each stage of the selection process and were granted access to one of the research facilities available in the MARINET consortium (rate of success $62.11 \%$ ). This in total corresponds to an equivalent 659 weeks of trials at the facilities for the various projects.

Such a large number of projects offers significant and valuable information constituting a database for assessment of the current status of research and development of marine renewable energy technology across Europe. It also provides an overview on the benefits and limitations of a transnational access programme such as this. Both aspects are presented and discussed in the following section. 


\section{Transnational Access data analysis}

Elements provided by applicants in the standard MARINET TA application form offer information on the status of research and development of technologies for marine renewable energies that extends beyond the technical and scientific aspects. The database, built from the 256 applications received in the five calls, is valuable in that regard and offers interesting insights on various trends such as, for instance, the distribution of marine renewable energy projects across Europe and the stage of development of the research and technology for this industry.

According to MARINET rules, the transnational access programme is open to all kinds of institutions including companies of any size, research centres, educational bodies, etc.

Table 4 below presents the number of eligible applications categorised by type of institution and gives an overview of the panel of user groups having applied in the various calls for proposals. More specifically, it shows that industry is largely represented, submitting the majority (about $2 / 3^{\text {rd }}$ ) of the applications. Over $50 \%$ of the applicants overall were SMEs. This figure also includes individual developers usually having limited access to research facilities. It should be pointed out that the marine renewable energy sector is also largely represented by research bodies, with over $37 \%$ of the projects proposed by universities or research centres. It should also be noted that this classification is based on the affiliation of the leader of the projects but that, as is often the case, the group of users in each project may be a consortium involving both industrial and academic partners.

\begin{tabular}{|l|c|c|}
\hline Type of Institution & Number of eligible applications & $\begin{array}{l}\text { \% of total number of } \\
\text { eligible applications }\end{array}$ \\
\hline Research Centre & 33 & 12.89 \\
\hline Educational body & 62 & 24.22 \\
\hline SMEs & 133 & 51.95 \\
\hline Large Company & 28 & 10.91 \\
\hline
\end{tabular}

Table 4: Number of applications per type of Institution

According to EU rules the applying groups (at least $50 \%$ of the members and including the leader) must work in an institution established in a Member State of the European Union (the EU-27) or a State associated to the EC's Framework Programme 7 and must work in a country other than the country where the legal entity operating the applied-for MARINET infrastructure is established.

The histogramme in figure 1 hereafter gives an overview of the distribution of applications by country of origin. Proposals were received from 21 countries across Europe (the EU-27 and FP7-associated countries). This represents a relatively high number showing interest in the development of marine renewable energy across Europe, even in countries with limited access to marine resources.

The United Kingdom and Ireland clearly stand out as the countries with the largest number of proposals, each with over 50 projects, followed by Spain with 30 proposals. These three countries together provided $53 \%$ of all the applications. 


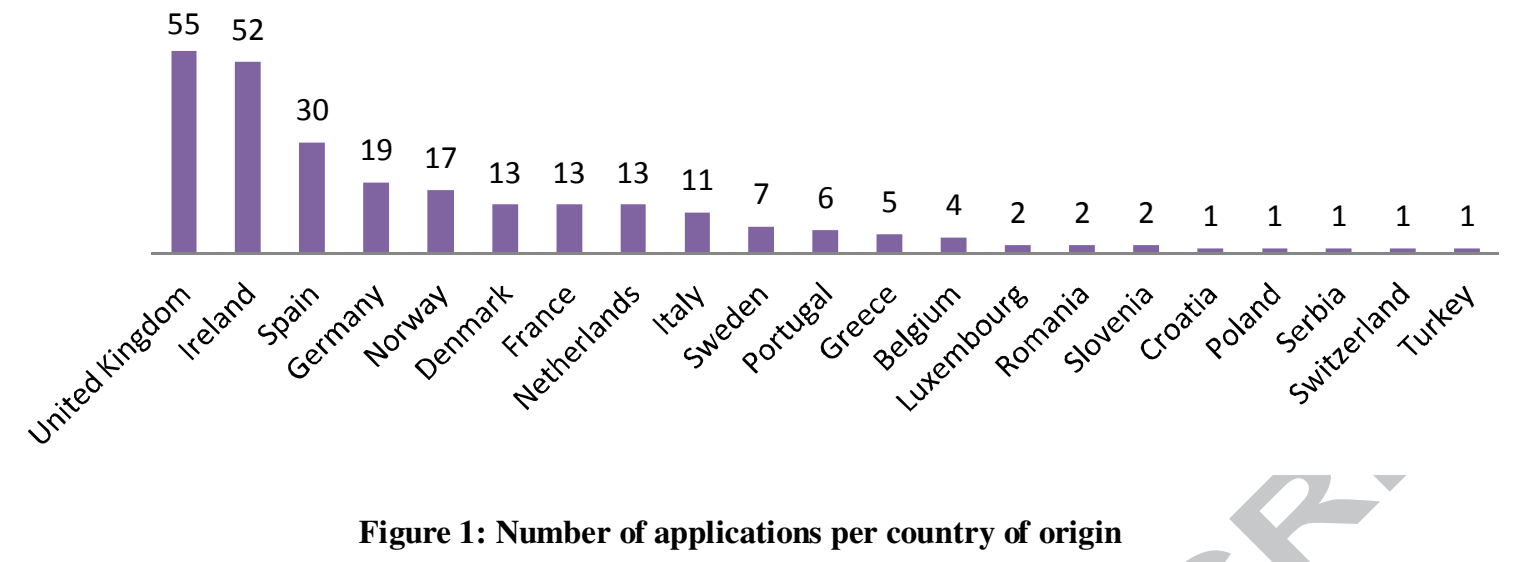

Facilities available in the MARINET consortium are spread across ten countries in Europe. The histogramme presented in figure 2 below shows a breakdown of the number of applications received per country over the five calls. It should be noted here that the $4^{\text {th }}$ call was a specific call open for access to a limited number of facilities related to cross-cutting and off-shore wind activities which had been in less demand in the previous calls, hence the lower number of applications for this particular call.

With a total of 153 proposals, France and the United Kingdom received about $59.8 \%$ of the total number of eligible projects, evenly distributed between the two countries. Italy, Ireland and Denmark follow with significantly less proposals $-27,24$ and 18 respectively. The five remaining countries gathered less than $15 \%$ of the proposals.

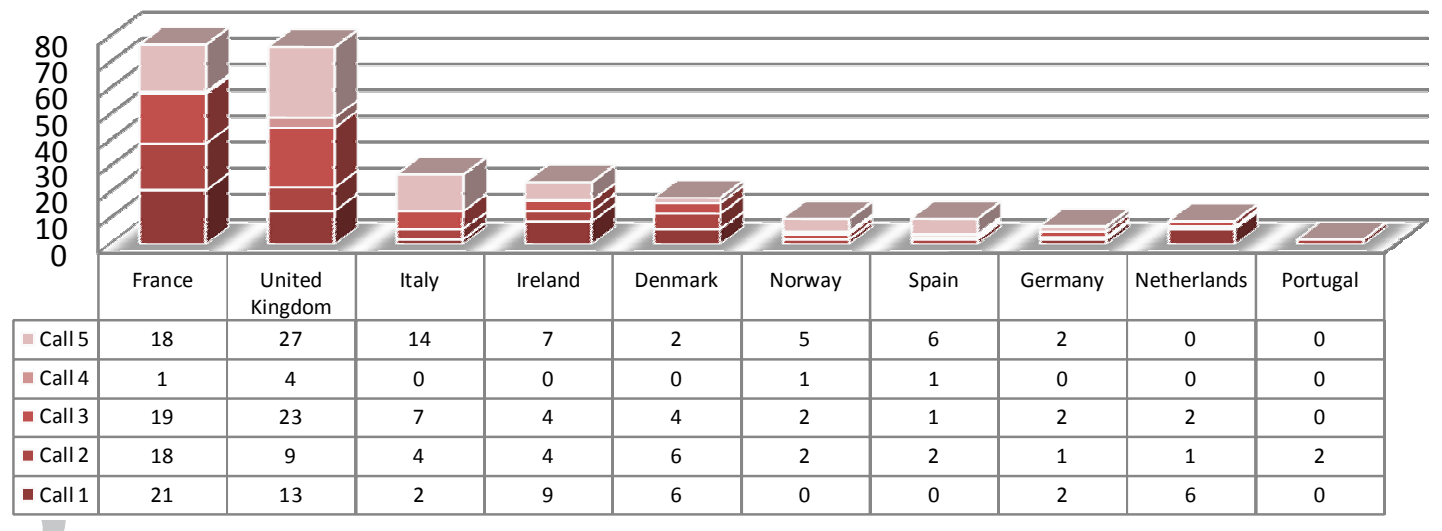

Figure 2: Number of eligible applications received per country over the 5 calls

Reasons for such a distribution are difficult to analyze as there are many parameters which need to be taken into account. The most obvious reason is the number of available facilities per country, but this does not provide a complete explanation. Other factors come into play, such as

- the testing scale of the infrastructures in each country (mid-development scale is in high demand),

- the discipline/specialty of the available infrastructures (certain specialist facilities are lower in demand as they are used at higher TRLs for example, e.g. electrical infrastructures) 
The number of infrastructures available per country within the MARINET consortium is given in figure 3. The United Kingdom offers the largest number of facilities, at thirteen, in all categories and scales of infrastructures. France provides four, as does Ireland, Germany and Italy which in total received the relatively lower figure of 58 proposals. All French facilities are in the "Large Lab" class, adapted for testing systems at stage 1 or 2, that is at TRL 1 to 4 . Even though these facilities are well known for their expertise and capabilities for MRE devices testing, for example the ECN HOET wave tank or the Ifremer current/wave flume, it should also be noted that they benefitted from the main eligibility rule stating that user groups must work in a country other than the country where the legal entity operating the applied-for MARINET infrastructure is established. They therefore received a large number of applications from both Ireland and the United Kingdom, countries with significant interest and activity in marine renewable energy development.

Adequacy and suitability of facilities for MRE device testing is a key element in the choice made by users. For instance the newly built Plymouth University COaST and Edinburgh University FLOWAVE facilities, offering capabilities of testing in both current and waves, were both added to the list of available facilities (large labs) only in calls 3 and 5 when they opened. Nonetheless both received a large number of applications (12 and 7 respectively). The high demand for these new facilities is also an indicator of the need for dedicated specialist research facilities.

Considering the countries that received fewer proposals, namely Norway, Spain, Germany, the Netherlands and Portugal, it should be noted that these countries offered a limited number of facilities (the largest being 4 facilities by Germany) and that at least $50 \%$ of those, in each country, are large or medium-scale testing sites adapted to testing of devices at an advanced development stage with a TRL 5 at the minimum. There are a significantly lower number of feasible projects test-ready at this TRL across Europe and even worldwide, compared to systems at the concept to design stage (TRL 1-4). It is significant and noteworthy, from this point of view, that two proposals were received by Wavec for the Portuguese Large Scale test site PICO OWC.

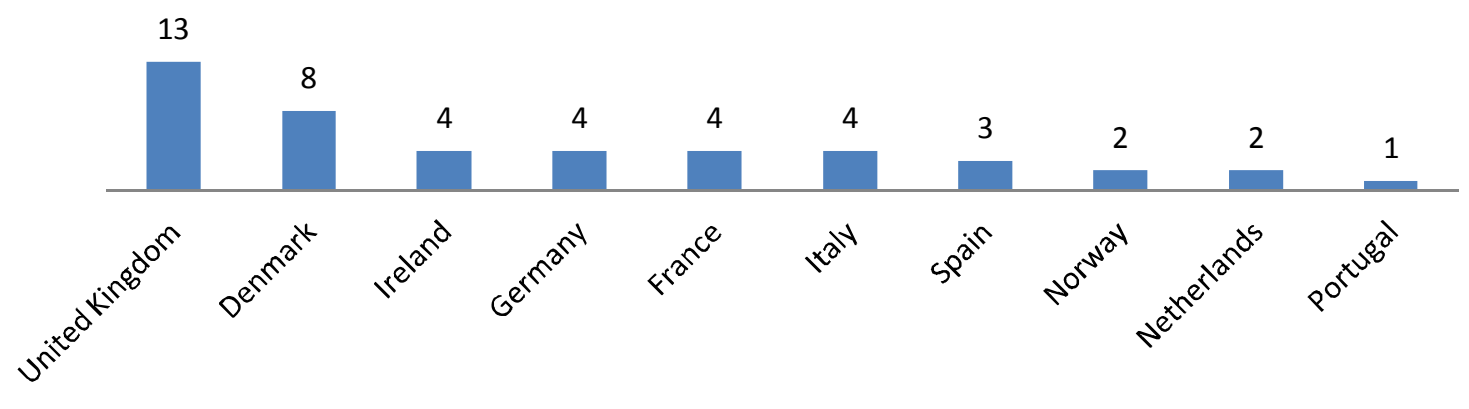

Figure 3: Number of available infrastructures per country

Since one of the objectives of MARINET is to coordinate research and development of MRE concepts at all scales it is also interesting to analyse the trends in the stage of development of the projects applying throughout the five calls organized over the duration of the project.

Table 5 below shows the number of applications received by the medium and large scale sites for each of the five calls. Excluding the $4^{\text {th }}$ call for which only a limited number of infrastructures were made available, the total number of advanced projects (TRL 5 or higher) per call seems to be rather stable, and ranging between 9 and 11. In fact, it can be observed that the ratio of these advanced projects over the total number of projects received per call decreases from $20 \%$ in call 1 to $13.6 \%$ in call 5 (except 
in call 4 for which the number of available testing sites in regard of the number of laboratories was larger). This trend seems to indicate that, in spite of the existence of adapted facilities, as well as technical and scientific support at disposal, it is difficult for developers to move from the design stage $($ TRL $<5)$ to more advanced stages.

Further illustrating this, and due to the fact that successful applicants could apply in a following call having completed a prior period of access, among the 256 applications in the various calls, a significant number were identified as corresponding to a previous concept development project. Considering these multi-phase projects, only a few of them, six, were identified as having applied to a large or medium scale test site, i.e. having reached Stage 3 (TRL 5-6), after completing a period of access in a MARINET laboratory in a previous call. Only three projects have actually been able to complete their advanced stage testing programme.

Various reasons could explain this low progression rate, which is nonetheless typical for marine renewable energy development generally. One is probably that the duration of the actual period of access to facilities within the Transnational Access programme, 30 months in total, is too short to allow an evolution from the stage of device optimization/performance verification at small scale to sea trials at large scale, involving concept analysis and optimization as well as prototype design and assembly. Economic factors should be considered as well, as the saving induced by the free-of-charge access to the testing site can be considered limited at larger scales compared to the cost of design and building of a large-scale prototype. The TA programme is thus less of an incentive for advanced projects. Finally it should be noted that a significant number of proposals were received for conducting several testing programmes of a same concept in various laboratories at the lower stages. This reflects the research and development reality of this sector whereby many small scale validation/optimization testing programmes are necessary by developers to improve the performance of their concept or of some parts of their concepts in order to pass a Stage gate and progress to the next Stage.

\begin{tabular}{|l|c|c|c|c|c|}
\hline & Call 1 & Call 2 & Call 3 & Call 4 & Call 5 \\
\hline Large Scale sites & 5 & 6 & 5 & 1 & 5 \\
\hline Medium scale sites & 6 & 3 & 5 & 2 & 6 \\
\hline Total & 11 & 9 & 10 & 3 & 11 \\
\hline \% of total number of projects per call & 20 & 18.4 & 15.6 & 42.8 & 13.6 \\
\hline
\end{tabular}

Table 5: Number of advanced projects per Call

Finally, considering the number of proposals received per focus area group, it can be noted that projects related to the wave energy sector were numerous, representing $42.2 \%$ of the whole number of eligible proposals. $20.3 \%$ of the projects were related to tidal energy whilst $14.5 \%$ were related to offshore-wind energy. Even though these figures do not take into account the kind of technology the cross-cutting focus group projects are related to (which is not always strictly defined), they provide some insight into the status of the various industries. In particular, the large number of wave energy related projects (108) with an abundance of technology concepts, most of them at an early stage of development, is in accordance with the relatively lesser mature stage of this sector.

\section{Benefits \& limitations of a TA programme}

Along with the necessary exchanges between users, infrastructure managers and the TA coordination group that took place as part of the selection and testing processes, networking activities were conducted over the course of the initiative with the main objective of fostering communication between users and MARINET partners. Specifically, workshops were organised as part of this activity 
(Consortium meeting at Boulogne-sur-Mer, France, October 2012 and Consortium/User-Group Workshop, Rome, Italy, November 2013) offering opportunities to obtain feedback from all parties involved in the TA programme. A further User-Group workshop will be held in 2015. Based on feedback from these meetings, the main benefits and limitations of such a TA programme are reported below.

\subsection{From the point of view of the visiting research group}

The main benefits of a TA programme for the research groups have been outlined above, and include:

- free-of-charge access to specialist infrastructures in a different country which may otherwise be difficult to access or be prohibitively expensive

- access to world-class specialist expertise of the personnel located at these infrastructures and throughout the MARINET network

- opportunity to avail of a structured test programme which can progress through linked infrastructures operating common standards, procedures and analysis methods

- validated test results from an infrastructure in a recognised programme such as MARINET which can be compared on a like-for-like basis with competing technologies

Research groups have reported perceived programme limitations such as:

- insufficient available access time to complete a full stage testing programme

- independent funding is needed to construct models or to purchase required instrumentation (in some instances) - the EU MARINET programme covers the test infrastructure costs, but not the model/device costs

- the requirement to use an infrastructure in a different country. For example, some UK researchers/developers have had issue with the fact that they are required to access an infrastructure outside their own country, when a suitable facility is already available in their own country. This misunderstands the purpose of an EU TA programme, which is to facilitate access for groups which do not have a specialised national infrastructure which they can access, both in terms of capabilities of a unique infrastructure and funding for such access outside the home country.

- the challenge to progress through the testing stages in the limited programme timeframe (4 years).

\subsection{From the point of view of the TA programme/Coordinator}

The benefits of a TA programme have largely been covered already in this paper. Suffice to say that the coordination of resources, expertise, standards and future European-wide infrastructure planning provides immense benefits to what would otherwise be a fragmented and inefficient provision of such services and facilities.

Some of the limitations or challenges of such a programme, and of coordinating such a programme, are outlined below:

- Short-notice cancellations by research groups mean a significant opportunity cost for the infrastructure involved. Due to the fact that the initiative/network is not a legal entity, deposits or other such disincentives are difficult to implement other than by infrastructures directly following their own usual procedures.

- Misunderstandings in relation to the purpose and operation of such a programme lead to unrealistic expectations as to what is being offered.

- It is difficult to balance the need to obtain sufficient information in order to make an informed decision about a research proposal, with the objective of keeping application forms simple, straightforward and accessible. Decisions on granted proposals are therefore a compromise between receiving sufficient information and ease of applying, and are heavily dependent on the feedback of the infrastructure manager who has been the main contact with the applicant.

- Independent evaluation of proposals is a challenge in an environment with a limited number of internal and external experts in a relatively close-knit sector, usually providing their expertise on a pro-bono basis. 
- Obtaining agreement on implementing testing standards, and ensuring implementation of those standards across the network of infrastructures, can be a challenge.

\section{Conclusions}

The MARINET initiative was started in March 2011 bringing together a network of 29 partners, spread across twelve countries to offer research groups access to 45 research facilities with the aim of streamlining the testing path to accelerate the development and commercial deployment of marine renewable energy technologies. The main tool developed for achieving this is the Transnational Access programme, offering free of charge access to research facilities for the testing of concepts for wave, tidal current and off-shore wind energy conversion as well as cross-cutting studies. Analysis of the metadata associated with the 256 eligible proposals that were received throughout the five calls that were organized over a period of 30 months provides an insight into the current status of research on marine renewable energy technologies across Europe, together with an overview of the benefits and limitations of such a Transnational Access programme.

Proposals were received from 21 countries across Europe, and the United Kingdom and Ireland were the most active with over 50 proposals each. Analysis of the distribution of the most in-demand facilities per development stage and per country indicates that most projects are at an early or intermediate stage of development, with a TRL of 1 to 4 , and that there is strong demand for specialist, dedicated research and testing facilities. It is also observed that, with such a programme having a limited duration, only a small number of well-organised projects have the ability to move from a validation/optimization stage requiring reduced scale laboratory testing to the stage of deployment of an operational device (TRL 5).

\section{Acknowledgement}

The work described in this paper has received support from the European Community's Research Infrastructure Action under the FP7 "Capacities" Specific Programme through grant agreement number 262552, MARINET.

\section{References}

[1] Healy M., Lewis T. and Alcorn R., 2012, “MARINET - Streamlined, free-of-charge access to test facilities: How world-class research centres are joining forces to accelerate the development of marine renewable energy", Proc. Of the 4th International Conference on Ocean Energy, ICOE2012, 17 October, Dublin, Ireland

[2] B. Holmes, K. Nielsen. (2010): Guidelines for the Development \& Testing of Wave Energy Systems. Report T02-2.1, OES-IA Annex II Task 2.1. Available: www.iea-oceans.org

[3] A.S. Bahaj, L.Blunden, A.A. Anwar. (2008): Tidal Energy Development Protocol. University of Southampton, Report from BERR for the OES-IA, OES-IA Annex II Task 2.2. Available: www.ieaoceans.org

[4] T. McCombes, C. Johnstone, B. Holmes, L.E. Myers, A.S. Bahaj, J.P. Kofoed. (2010): Best Practice for Tank Testing of Small Marine Energy Devices. EC FP7 EquiMar (Grant Agreement 213380), Deliverable D3.4. Available: www.equimar.org. 
- The Transnational Access programme : a key element of the MARINET initiative

- 256 proposals providing a good overview of the stage of development of the Marine Renewable Energy industry in Europe 